Cite this: Org. Biomol. Chem., 2014, 12,307

Received 3rd September 2013, Accepted 7th November 2013

DOI: $10.1039 / c 3 o b 41798 k$

www.rsc.org/obc

\section{The acid-catalysed synthesis of 7-azaindoles from 3-alkynyl-2-aminopyridines and their antimicrobial activity $\dagger$}

\author{
Tlabo C. Leboho, ${ }^{a}$ Sandy F. van Vuuren, ${ }^{\mathrm{b}}$ Joseph P. Michael ${ }^{\mathrm{a}}$ and \\ Charles B. de Koning*a
}

The synthesis of 7-azaindoles from 3-alkynyl-2-aminopyridines using acidic conditions, namely, a mixture of trifluoroacetic acid (TFA) and trifluoroacetic anhydride (TFAA), is described. This methodology resulted in the synthesis of fifteen 7-azaindoles, with most containing substituents at the 2 - and 5 -positions. The majority of these were tested for antimicrobial activity against a range of bacteria and yeasts. The 7-azaindoles displayed the best activity against the yeasts, particularly against Cryptococcus neoformans, where activities as low as $3.9 \mathrm{\mu g} \mathrm{ml}^{-1}$ were observed.

\section{Introduction}

The azaindoles are a growing class of indole isosteres that possess promising biological profiles. For example, the natural product variolin B 1 (Fig. 1) isolated from an extremely rare Antarctic sponge is a promising anti-cancer agent. ${ }^{1}$

Other examples include the synthetic azaindole derivative of rebeccamycin, known as diazarebeccamycin 2, which has also been shown to exhibit promising anticancer activity, ${ }^{2}$ and 6-azaindole 3 which has demonstrated activity as an inhibitor of HIV-1 attachment to $\mathrm{CD}_{4}{ }^{+}$T cells. ${ }^{3}$

The synthesis of 7-azaindoles is often accomplished by initial Sonogashira coupling of a 3-halosubstituted-2-aminopyridine as shown in Scheme $1{ }^{4}$ The intermediate alkynylpyridines are then exposed to a variety of different reaction conditions and reagents to afford the desired azaindoles. Many methods have been developed for this step and include the use of cesium- or potassium-containing bases such as potassium hydride, potassium $t$-butoxide or cesium $t$-butoxide ${ }^{5 a-d}$ or even bases such as $\mathrm{DBU}^{5 e}$ and triethylamine. ${ }^{5 f}$ Metalcontaining catalysts have also been used for this purpose and include amongst others, the use of copper, ${ }^{6 a}$ an indium salt ${ }^{6 b}$ or even gold catalysts. ${ }^{6 c}$ The Cacchi reaction in the presence of a palladium catalyst ${ }^{6 d}$ has also been successfully used to facilitate both the Sonogashira coupling reaction and the azaindole

${ }^{a}$ Molecular Sciences Institute, School of Chemistry, University of the Witwatersrand, PO Wits 2050, Johannesburg, South Africa.E-mail: Charles.deKoning@wits.ac.za; Fax: +27 11 7176749; Tel: +27 117176724

${ }^{b}$ Department of Pharmacy and Pharmacology, Faculty of Health Sciences, University of the Witwatersrand, Johannesburg, South Africa

$\dagger$ Electronic supplementary information (ESI) available: Copies of ${ }^{1} \mathrm{H}$ and ${ }^{13} \mathrm{C}$ NMR spectra are provided. See DOI: 10.1039/c3ob41798k<smiles></smiles><smiles></smiles><smiles>COc1cnc(-n2cnc(C)n2)c2[nH]cc(C(=O)C(=O)N3CCN(/C(C)=C\c4ccccc4)CC3)c12</smiles>

Diazarebeccamycin 2

Fig. 1 Examples of biologically active azaindoles.
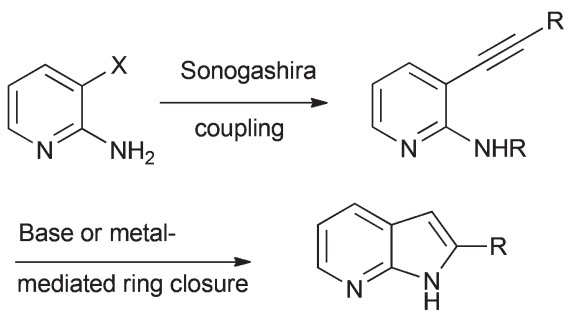

Scheme 1 
ring forming step to produce 2,3-disubstituted azaindoles directly from the aminopyridines. Even the use of microwaves in the presence of water with ${ }^{7 a}$ or without ${ }^{7 b}$ added salts has been reported.

As a result of our interest in the synthesis of indole-containing compounds and their antibacterial and antifungal activity, ${ }^{8}$ we initiated a programme directed towards the synthesis of the related compounds, azaindoles. The specific goal described in this publication was to attempt to develop versatile and cheap conditions for the synthesis of 7-azaindoles from the corresponding Sonogashira coupled 3-alkynyl2-aminopyridine precursors. Thereafter, as azaindoles have interesting biological profiles, we wished to ascertain if the 7-azaindoles we had synthesized would display activity against a range of bacterial and yeast cell lines.

\section{Results and discussion}

Initially the required halogenated substituted pyridines were prepared or obtained from commercial sources. 5-Chloro2-aminopyridine 4, 2-aminopyridine 5, 3-bromo-2-aminopyridine 6 and 5-methyl-2-amino-3-bromopyridine 7 were obtained from Sigma-Aldrich. 5-Chloro-2-aminopyridine 4 was treated with $\mathrm{I}_{2}$ in the presence of $\mathrm{Ag}_{2} \mathrm{SO}_{4}{ }^{9}$ in ethanol to afford the 3 -iodo substituted aminopyridine $\mathbf{8}$ in good yield.

Finally, 5-bromo-3-iodopyridin-2-amine 10 was prepared by initially treating 2-aminopyridine 5 with $\mathrm{NBS}^{10}$ in $\mathrm{MeCN}$ to afford 5-bromo-2-aminopyridine 9. Then 9 was subjected to potassium iodate, $\mathrm{I}_{2}$ and a mixture of $\mathrm{H}_{2} \mathrm{SO}_{4}$, acetic acid and water $^{6 a}$ to provide 5-bromo-3-iodopyridin-2-amine 10 in good yield (Scheme 2).

We were now in a position to attempt the Sonogashira coupling reaction, for which we believed that coupling would take place at the more reactive iodine substituent in substrates $\mathbf{8}$ and 10, or at the bromine in the case of aminopyridines 6 and 7. The 2-amino-3-halogen containing pyridines were treated with catalytic tetrakis(triphenylphosphine)palladium(0), copper(I) iodide, triethylamine as a base and a range of acety lenes to afford the desired compounds (11-14) in good to excellent yield as shown in Scheme 3.

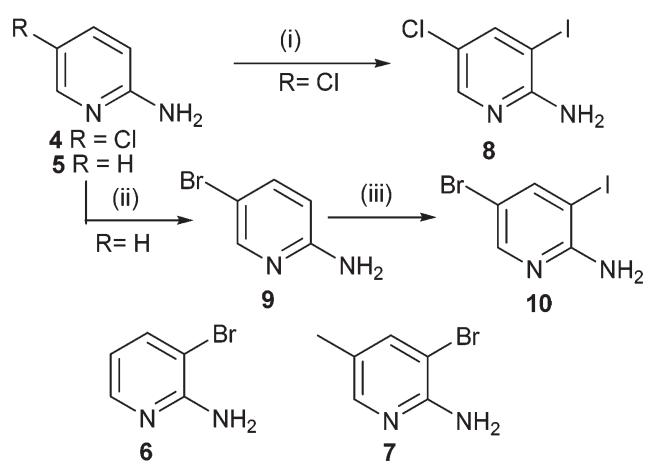

Scheme 2 Reagents and conditions: (i) $\mathrm{I}_{2}, \mathrm{Ag}_{2} \mathrm{SO}_{4}, \mathrm{EtOH}, \mathrm{rt}, 86 \%$; (ii) NBS, MeCN, rt, $98 \%$; (iii) $\mathrm{I}_{2}, \mathrm{KIO}_{3}, \mathrm{H}_{2} \mathrm{SO}_{4}, \mathrm{AcOH}, \mathrm{H}_{2} \mathrm{O}, 80^{\circ} \mathrm{C}, 90 \%$.
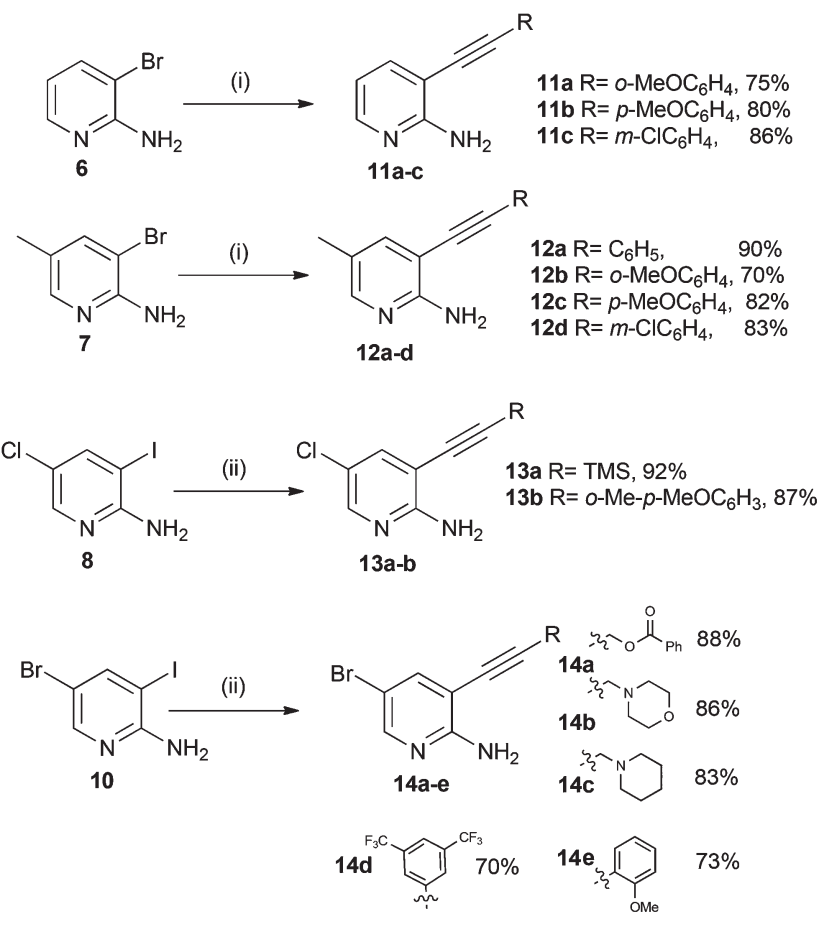

Scheme 3 Reagents and conditions: (i) $\mathrm{RC} \equiv \mathrm{CH}_{1} \mathrm{Et}_{3} \mathrm{~N}, \mathrm{Cul}$, cat. Pd$\left(\mathrm{PPh}_{3}\right)_{4}, \mathrm{THF}, 70^{\circ} \mathrm{C}$; (ii) $\mathrm{RC} \equiv \mathrm{CH} \mathrm{Et}_{3} \mathrm{~N}, \mathrm{Cul}$, cat. $\mathrm{Pd}\left(\mathrm{PPh}_{3}\right)_{4}, \mathrm{THF}$, rt.

The stage was now set to attempt the formation of the pyrrole ring in order to assemble the 7-azaindole nucleus. For this step we wished to investigate if it was possible to use acidic conditions to affect the ring closure. A variety of acids $\left(\mathrm{HCl}, \mathrm{H}_{2} \mathrm{SO}_{4}, \mathrm{AcOH}\right.$ and trifluoroacetic acid (TFA)) were initially used. The use of TFA seemed to be promising as a small amount of azaindole was forming but the conversion rate was estimated to be only $10 \%$. Since there are examples in the literature $^{4}$ of the corresponding amide (rather than amine) undergoing ring closure to afford azaindoles, we thought that the addition of trifluoroacetic anhydride (TFAA) to the reaction mixture containing TFA might improve the rate, conversion and yield of the desired azaindoles. After much experimentation we found that in the presence of 1 equivalents of TFA and 1.3 equivalents of TFAA in MeCN as a solvent and heating to reflux for $8 \mathrm{~h}$, the substrate afforded the desired azaindoles (15-18) in generally excellent yields (Scheme 4).

As one final example we wished to take advantage of the different reactivity of the halogens on substrate 10 to establish if it was possible to introduce two different alkyne substituents using different temperatures. Hence as shown in Scheme 5, 10 was treated under Sonogashira reaction conditions with phenylacetylene initially at room temperature. After TLC showed that the starting material had all been consumed, the solvent was evaporated under reduced pressure and the crude material was then subjected to the same palladium catalyst using trimethylsilylacetylene as the alkyne coupling partner. After the reaction was deemed to be complete by TLC, work-up of the reaction and analysis of the product by NMR spectroscopy showed that two different alkyne substituents were present on 
<smiles>[R]C#Cc1cccnc1N</smiles>

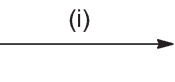

11a $\mathrm{R}=0-\mathrm{MeOC}_{6} \mathrm{H}_{4}, 11 \mathrm{a}$ 11b $\mathrm{R}=p-\mathrm{MeOC}_{6} \mathrm{H}_{4}, 11 \mathrm{~b}$ 11c $\mathrm{R}=m-\mathrm{ClC}_{6} \mathrm{H}_{4}, 11 \mathrm{c}$<smiles>[R]C#Cc1cc(C)cnc1N</smiles>
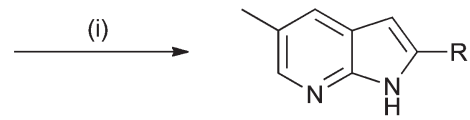

12a $\mathrm{R}=\mathrm{C}_{6} \mathrm{H}_{5}$ $12 \mathrm{~b} \mathrm{R}=0-\mathrm{MeOC}_{6} \mathrm{H}_{4}$ 12c $\mathrm{R}=p-\mathrm{MeOC}_{6} \mathrm{H}_{4}$ $12 \mathrm{~d} \mathrm{R}=m-\mathrm{ClC}_{6} \mathrm{H}_{4}$<smiles>[R]C#Cc1cc(Cl)cnc1N</smiles><smiles>[R]c1cc2cc(Cl)cnc2[nH]1</smiles>

13a $\mathrm{R}=\mathrm{TMS}$ 13b R= o-Me-p-MeOC ${ }_{6} \mathrm{H}_{3}$

$17 \mathrm{a} \mathrm{R}=\mathrm{H}$

$84 \%$
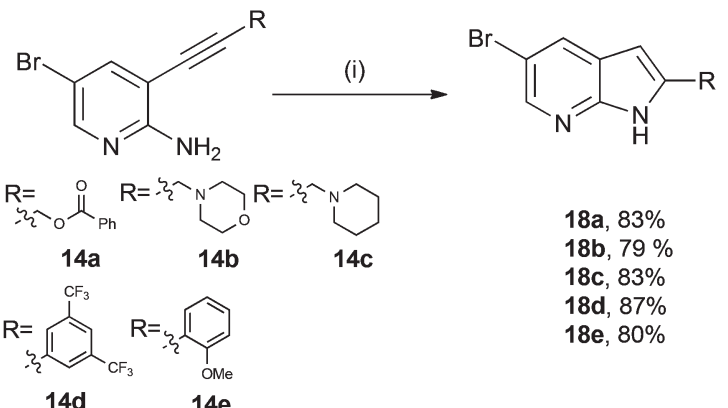

$18 \mathrm{a}, 83 \%$ 18b, $79 \%$ $18 \mathrm{c}, 83 \%$ 18d, $87 \%$ $18 \mathrm{e}, 80 \%$

Scheme 4 Reagents and conditions: (i) TFA, TFAA, MeCN, $100^{\circ} \mathrm{C}$.

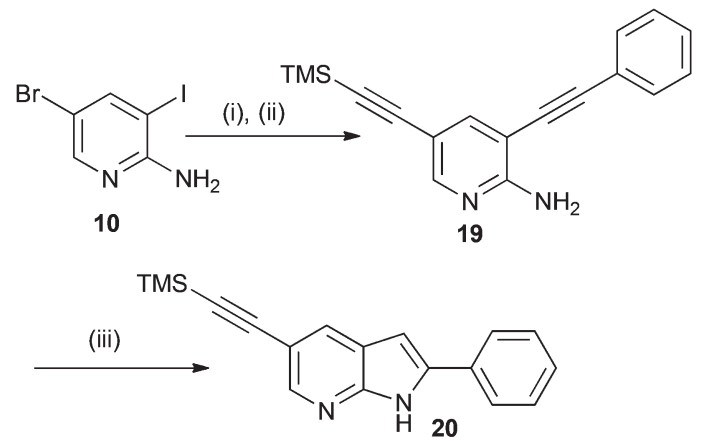

Scheme 5 Reagents and conditions: (i) $\mathrm{Ph}-\mathrm{C} \equiv \mathrm{CH}, \mathrm{Et}_{3} \mathrm{~N}, \mathrm{Cul}, \mathrm{Pd}-$ $\left(\mathrm{PPh}_{3}\right)_{4}, \mathrm{THF}, \mathrm{rt}$; (ii) $\mathrm{TMS}-\mathrm{C} \equiv \mathrm{CH}, \mathrm{Et}_{3} \mathrm{~N}, \mathrm{Cul}, \mathrm{Pd}\left(\mathrm{PPh}_{3}\right)_{4}, \mathrm{THF}, 70{ }^{\circ} \mathrm{C} .85 \%$ over two steps; (iii) TFA, TFAA, MeCN, $100{ }^{\circ} \mathrm{C}, 91 \%$.

the 3,5-substituted aminopyridine 19. Presumably, this is due to the different reactivities of the iodine and bromine substituents in the two Sonogashira reactions. Exposure of $\mathbf{1 9}$ to our acid mediated reaction conditions for azaindole formation resulted in the formation of the desired azaindole 20 in excellent yield.

As we now had a series of substituted azaindoles on hand they were quantitatively evaluated for antimicrobial activity
(Table 1). While compound 16b showed promising antibacterial activity $\left(7.8 \mu \mathrm{g} \mathrm{ml}^{-1}\right)$ against Pseudomonas aeruginosa, many of the azaindoles showed very good and selective anti-yeast activity, particularly against Cryptococcus neoformans, where all compounds showed noteworthy inhibitory values of between 3.9-15.6 $\mu \mathrm{g} \mathrm{ml}^{-1}$. Previous studies on the antimicrobial effects of other azaindole derivatives include the quantitative study by Kumaran et al. ${ }^{11}$ and the promising antifungal effects found by Lebouvier. ${ }^{12}$ Previous antimicrobial studies on azaindoles for activity against Cryptococcus neoformans is, however, lacking and the promising activity noted here warrants further examination, particularly, given that increased reports of infections from Cryptococcus neoformans show that this represents a major health threat for HIV infected patients. ${ }^{13,14}$ Newer antifungals to combat this opportunistic pathogen will certainly be beneficial in future treatments, particularly with emerging trends of resistance evident.

In summary, to the best of our knowledge this is the first report outlining the synthesis of azaindoles from 3-alkynyl2 -aminopyridines under acidic conditions. The azaindoles synthesized display significant anti-yeast activity.

\section{Experimental}

${ }^{1} \mathrm{H}$ NMR and ${ }^{13} \mathrm{C}$ NMR spectra were recorded either on a Bruker AVANCE 300 spectrometer or a Bruker AVANCE III 500 spectrometer. All chemical shift values are reported in parts per million referenced against TMS which is given an assignment of zero parts per million. Coupling constants ( $J$-values) are given in hertz $(\mathrm{Hz})$. All mass spectroscopy data were collected on a Waters Acquity UPLC system coupled to a Waters HDMS G1 QTOF mass spectrometer. UPLC settings: analytical column: BEH C18 $150 \times 2.1 \mathrm{~mm}$; column temperature: $60{ }^{\circ} \mathrm{C}$; mobile phase: $90 \%$ water $(0.1 \%$ formic acid): $5 \%$ acetonitrile; flow rate: $0.4 \mathrm{ml} \mathrm{min}^{-1}$. MS settings: mode: VTOF; ionisation: ESIPos and ESINeg; scan range: 100-1000 Da; scan speed: 0.1 second; run time: $10 \mathrm{~min}$. Infrared spectra were recorded on a Bruker Tensor 27 standard system spectrometer with diamond ATR attachment. Macherey-Nagel Kieselgel 60 (particle size $0.063-0.200 \mathrm{~mm}$ ) was used for conventional silica gel column chromatography with various EtOAc and hexane mixtures as the mobile phase. TLC was performed on aluminum-backed Macherey-Nagel Alugram Sil G/UV254 plates pre-coated with $0.25 \mathrm{~mm}$ silica gel 60 . The following acetylenes are commercially available: phenylacetylene, trimethylsilylacetylene, 2-ethynylanisole, 4-ethynylanisole, 1-ethynyl-4-methoxy-2methylbenzene, 1-ethynyl-3,5-bis(trifluoromethyl)benzene and propargylbenzoate. The remaining two acetylenes 1-(prop-2ynyl)piperidine and 4-(prop-2-ynyl)morpholine were prepared according to ref. 15 and 16.

\section{5-Chloro-3-iodo-2-aminopyridine 8}

To a solution of 2-amino-5-chloropyridine $4(0.700 \mathrm{~g}$, $5.45 \mathrm{mmol}$ ) in ethanol was added silver sulfate $(1.699 \mathrm{~g}$, 
Table 1 Antimicrobial and antifungal activity of selected azaindoles $\left(\mu \mathrm{g} \mathrm{ml}^{-1}\right)$

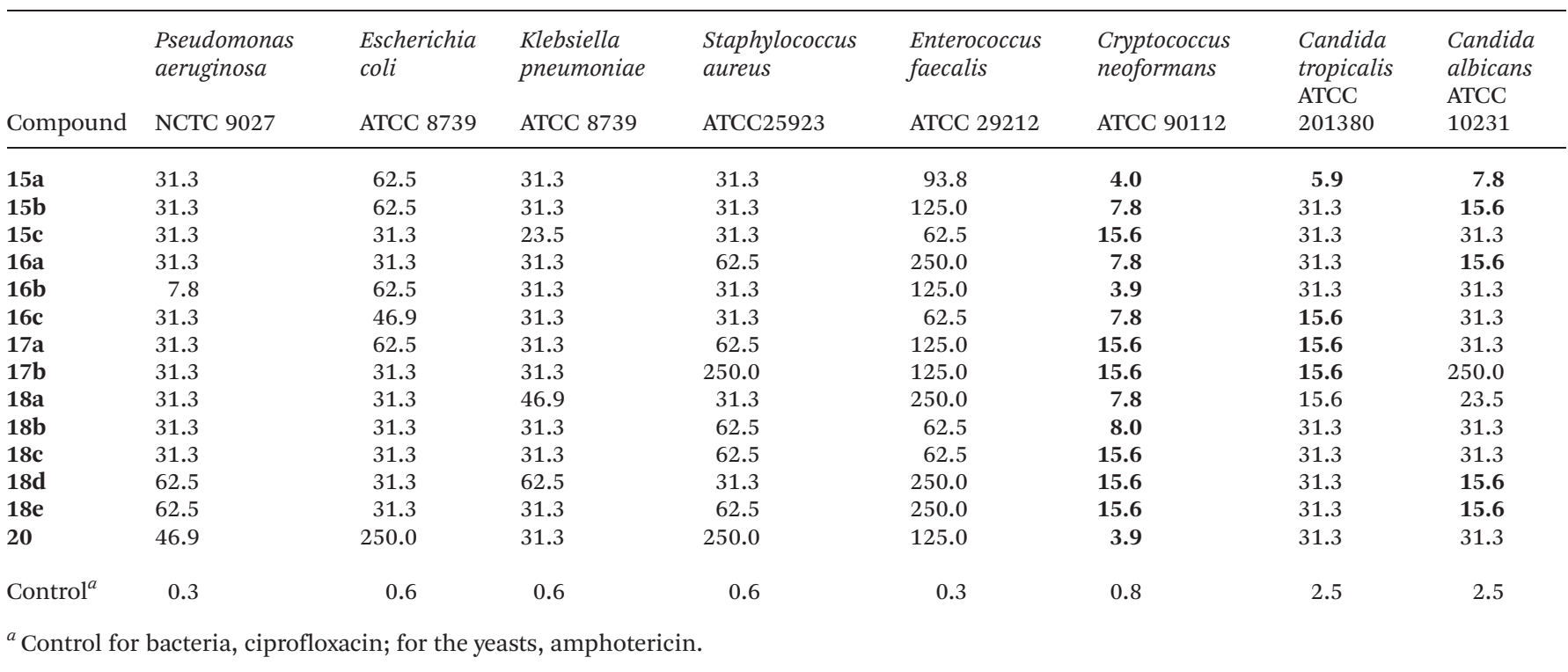

$5.45 \mathrm{mmol}$ ) in one portion followed by the addition of iodine (1.383 g, $5.45 \mathrm{mmol}$ ) portionwise. The heterogeneous mixture was stirred at rt until determined complete by TLC. After the reaction was complete, the mixture was filtered through celite or silica gel and the ethanol removed under reduced pressure. The resulting crude material was re-dissolved in dichloromethane and washed with a saturated solution of aqueous sodium thiosulfate. The separated dichloromethane layer was removed under reduced pressure and the resulting solid was purified by silica gel chromatography to give 2-amino-5-chloro3-iodopyridine $8^{9}$ (1.189 g 86\%). ${ }^{1} \mathrm{H}$ NMR (300 $\mathrm{MHz}, \mathrm{CDCl}_{3}$ ) $\delta 7.98(\mathrm{~d}, J=2.2,1 \mathrm{H}), 7.84(\mathrm{~d}, J=2.2,1 \mathrm{H}), 5.06(\mathrm{~s}, 2 \mathrm{H})$.

\section{5-Bromo-2-aminopyridine 9}

2-Aminopyridine 5 (2.600 g, $0.0276 \mathrm{~mol}$ ) was dissolved in dry MeCN (100 ml), treated with $N$-bromosuccinimide $(5.219 \mathrm{~g}$, $0.0293 \mathrm{~mol}$ ) and stirred for $2 \mathrm{~h}$ at rt. The solvent was removed and the resulting cream white solid purified by flash chromatography to give 2-amino-5-bromopyridine $\mathbf{9}^{10}$ as a white solid (4.692 g, 98\%). ${ }^{1} \mathrm{H}$ NMR (300 MHz, $\mathrm{CDCl}_{3}$ ) $\delta 8.08$ (s, 1H), 7.48 $(\mathrm{dd}, J=8.7,2.4,1 \mathrm{H}), 6.41(\mathrm{~d}, J=8.7,1 \mathrm{H}), 4.44(\mathrm{~s}, 2 \mathrm{H}) ;{ }^{13} \mathrm{C} \mathrm{NMR}$ $\left(75 \mathrm{MHz} \mathrm{CDCl}_{3}\right) \delta 157.36,148.88,140.49,110.42,108.56$.

\section{5-Bromo-3-iodo-2-aminopyridine 10}

A mixture of 2-amino-5-bromopyridine 9 (45.00 g, $0.260 \mathrm{~mol})$, iodic acid (11.86 g, $0.0676 \mathrm{~mol})$, iodine $(26.40 \mathrm{~g}, 0.104 \mathrm{~mol})$, sulfuric acid $(4.50 \mathrm{ml})$, acetic acid $(150 \mathrm{ml})$ and water $(30 \mathrm{ml})$ was heated to $80{ }^{\circ} \mathrm{C}$ for $8 \mathrm{~h}$. After this time, the reaction mixture was concentrated under vacuum and then made basic with an aqueous $12 \mathrm{M}$ sodium hydroxide solution. The basic solution was extracted with dichloromethane, washed with a saturated solution of sodium thiosulfate and then brine, dried over $\mathrm{MgSO}_{4}$ and then the organic solvent was removed under vaccum. The resulting cream white solid was purified by flash chromatography to give 2-amino-5-bromo-3-iodopyridine $\mathbf{1 0}^{6 a}$ as a white solid (70.11 g, 90\%). ${ }^{1} \mathrm{H}$ NMR (300 $\mathrm{MHz}, \mathrm{CDCl}_{3}$ ) $\delta 8.05(\mathrm{~s}, 1 \mathrm{H}), 7.95(\mathrm{~s}, 1 \mathrm{H}), 5.03(\mathrm{~s}, 2 \mathrm{H}) ;{ }^{13} \mathrm{C} \mathrm{NMR}(75 \mathrm{MHz}$, $\left.\mathrm{CDCl}_{3}\right) \delta 156.75,148.72,148.57,107.58,77.93$.

\section{General procedure for the Sonogashira reaction to synthesize 11a-c and 12a-d}

To a flame-dried round-bottom flask under a nitrogen or argon atmosphere containing 2-amino-3-bromopyridine derivative (6 and 7) was added CuI ( $2 \mathrm{~mol} \%)$ and $\mathrm{Pd}\left(\mathrm{PPh}_{3}\right)_{4}(2 \mathrm{~mol} \%)$ in one portion followed by the addition of the degassed alkyne solution in THF $(20 \mathrm{ml})$. Triethylamine $(5 \mathrm{ml})$ was then added and the reaction mixture was stirred at $70{ }^{\circ} \mathrm{C}$ until no starting material was present as monitored by thin layer chromatography (TLC). After this time, the reaction was quenched with a saturated aqueous ammonium chloride and was then extracted with either dichloromethane or ethyl acetate. The combined organic extracts were dried over magnesium sulfate, filtered through either silica or celite and the excess solvent was removed on a rotary evaporator, followed by purification using flash chromatography (30\% EtOAc-hexane) to yield the desired products $\mathbf{1 1 a - c}$ and 12a-d.

3-[(2-Methoxyphenyl)ethynyl]pyridin-2-amine 11a. Using 2-amino-3-bromopyridine (0.450 g, $2.60 \mathrm{mmol})$, 1-ethynyl2-methoxybenzene (0.343 g, $2.60 \mathrm{mmol}), \mathrm{CuI}$ (9.91 mg, $0.0520 \mathrm{mmol})$ and $\mathrm{Pd}\left(\mathrm{PPh}_{3}\right)_{4}(0.0612 \mathrm{~g}, 0.0520 \mathrm{mmol})$ the product 11a was obtained as a cream white solid $(0.351 \mathrm{~g}$, 75\%). Mp: $118-120{ }^{\circ} \mathrm{C} ;{ }^{1} \mathrm{H}$ NMR (500 MHz, $\left.\mathrm{CDCl}_{3}\right) \delta 8.03$ (d, $J=5.0,1.6,1 \mathrm{H}), 7.57(\mathrm{dt}, J=13.5,6.7,1 \mathrm{H}), 7.46(\mathrm{dd}, J=7.6$, 1.6, $1 \mathrm{H}), 7.32(\mathrm{td}, J=8.4,1.7,1 \mathrm{H}), 6.98-6.89$ (m, 2H), 6.62 (dd, $J=7.5,5.0,1 \mathrm{H}), 5.34(\mathrm{~s}, 2 \mathrm{H}), 3.92(\mathrm{~s}, 3 \mathrm{H}) ;{ }^{13} \mathrm{C}$ NMR $(126 \mathrm{MHz}$, $\left.\mathrm{CDCl}_{3}\right) \delta 159.92,159.17,147.83,138.83,132.44,129.95,120.65$, 113.27, 112.09, 110.53, 103.46, 92.26, 89.30, 55.82. IR $\left(\mathrm{cm}^{-1}\right)$ 3298 (NH str.), 2997 (CH str.), 2312 (alkyne), $1619(\mathrm{C}=\mathrm{N}), 1580$ 
$(\mathrm{C}=\mathrm{C}), 1181(\mathrm{C}-\mathrm{O})$. HRMS $\left(\mathrm{ES}^{+}\right)$Calculated for $\mathrm{C}_{14} \mathrm{H}_{13} \mathrm{~N}_{2} \mathrm{O}$ $[\mathrm{M}+\mathrm{H}]^{+}$: 225.1028, found 225.1032.

3-[(4-Methoxyphenyl)ethynyl]pyridin-2-amine 11b. Using 2-amino-3-bromopyridine $(0.450 \mathrm{~g}, 2.60 \mathrm{mmol})$, 1-ethynyl4-methoxybenzene (0.350 g, $2.60 \mathrm{mmol})$, CuI (9.91 mg, $0.0520 \mathrm{mmol})$ and $\mathrm{Pd}\left(\mathrm{PPh}_{3}\right)_{4}(0.0612 \mathrm{~g}, 0.0520 \mathrm{mmol})$ the product 3-[(4-methoxyphenyl)ethynyl]pyridin-2-amine 11b (0.389 $\mathrm{g}, 80 \%)$ was obtained as a cream white solid. Mp: 159-161 ${ }^{\circ} \mathrm{C} ;{ }^{1} \mathrm{H}$ NMR $\left(500 \mathrm{MHz}, \mathrm{CDCl}_{3}\right) \delta 8.02$ (dd, $J=4.9,1.5$, $1 \mathrm{H}), 7.57$ (dd, $J=7.5,1.7,1 \mathrm{H}), 7.50-7.41(\mathrm{~m}, 2 \mathrm{H}), 6.93-6.83$ $(\mathrm{m}, 2 \mathrm{H}), 6.63(\mathrm{dd}, J=7.5,5.0,1 \mathrm{H}), 5.05(\mathrm{~s}, 2 \mathrm{H}), 3.83(\mathrm{~s}, 3 \mathrm{H})$; ${ }^{13} \mathrm{C}$ NMR $\left(126 \mathrm{MHz}, \mathrm{CDCl}_{3}\right) \delta 159.93,158.75,147.69,139.71$, 133.01, 114.79, 114.13, 113.57, 103.54, 95.49, 83.14, 55.34; IR $\left(\mathrm{cm}^{-1}\right) 3245$ (NH str.), 2986 (CH str.), 2359 (alkyne), 1619 $(\mathrm{C}=\mathrm{N}), 1558(\mathrm{C}=\mathrm{C}), 1144(\mathrm{C}-\mathrm{O}) . \mathrm{HRMS}\left(\mathrm{ES}^{+}\right)$Calculated for $\mathrm{C}_{14} \mathrm{H}_{13} \mathrm{~N}_{2} \mathrm{O}[\mathrm{M}+\mathrm{H}]^{+}$: 225.1028, found 225.1039.

3-[(3-Chlorophenyl)ethynyl]pyridin-2-amine

11c. Using 2-amino-3-bromopyridine $(0.450 \mathrm{~g}, 2.60 \mathrm{mmol})$, 1-ethynyl3-chlorobenzene (0.354 g, $2.60 \mathrm{mmol})$, CuI (9.91 mg, $0.0520 \mathrm{mmol})$ and $\mathrm{Pd}\left(\mathrm{PPh}_{3}\right)_{4}(0.0612 \mathrm{~g}, 0.0520 \mathrm{mmol})$ the product 11c $(0.373 \mathrm{~g}, 86 \%)$ was obtained as a cream white solid. Mp: $117-120{ }^{\circ} \mathrm{C} ;{ }^{1} \mathrm{H}$ NMR $\left(500 \mathrm{MHz}, \mathrm{CDCl}_{3}\right) \delta 8.06$ (s, $1 \mathrm{H}), 7.60(\mathrm{~d}, J=7.3,1 \mathrm{H}), 7.51(\mathrm{~s}, 1 \mathrm{H}), 7.40(\mathrm{~d}, J=7.2,1 \mathrm{H})$, 7.37-7.27 (m, 2H), $6.67(\mathrm{~s}, 1 \mathrm{H}), 5.10(\mathrm{~s}, 2 \mathrm{H}) ;{ }^{13} \mathrm{C} \quad \mathrm{NMR}$ $\left(126 \mathrm{MHz}, \mathrm{CDCl}_{3}\right) \delta 158.72,148.28,140.23,134.37,131.34$, 129.72, 129.61, 128.91, 124.40, 113.66, 102.65, 94.04, 85.60; IR $\left(\mathrm{cm}^{-1}\right) 3326$ (NH str.), 3003 (CH str.), 2219 (alkyne), 1627 $(\mathrm{C}=\mathrm{N}), 1565(\mathrm{C}=\mathrm{C}), 1198(\mathrm{C}-\mathrm{O})$; HRMS $\left(\mathrm{ES}^{+}\right)$Calculated for $\mathrm{C}_{13} \mathrm{H}_{10} \mathrm{~N}_{2} \mathrm{Cl}[\mathrm{M}+\mathrm{H}]^{+}$: 229.0533, found 229.0542.

5-Methyl-3-(phenylethynyl)pyridin-2-amine 12a. Starting with 2-amino-3-bromo-5-methylpyridine (0.450 g, $2.41 \mathrm{mmol})$, phenylacetylene $(0.2455 \mathrm{~g}, 2.41 \mathrm{mmol}), \mathrm{CuI}(9.18 \mathrm{mg}$, $0.0482 \mathrm{mmol})$ and $\mathrm{Pd}\left(\mathrm{PPh}_{3}\right)_{4}(0.0557 \mathrm{~g}, 0.0482 \mathrm{mmol})$ the product $12 \mathrm{a}^{5 c}(0.450 \mathrm{~g}, 90 \%)$ was obtained as a cream white solid. ${ }^{1} \mathrm{H}$ NMR $\left(300 \mathrm{MHz}, \mathrm{CDCl}_{3}\right) \delta 7.90(\mathrm{~d}, J=1.6,1 \mathrm{H})$, 7.57-7.49 (m, 2H), 7.47 (d, $J=1.9,1 \mathrm{H}), 7.38$ (dd, $J=6.5,2.7$, $3 \mathrm{H}), 4.97(\mathrm{~s}, 2 \mathrm{H}), 2.21(\mathrm{~s}, 3 \mathrm{H}) ;{ }^{13} \mathrm{C} \mathrm{NMR}\left(75 \mathrm{MHz}, \mathrm{CDCl}_{3}\right.$ ) $\delta$ 156.86, 147.82, 140.62, 131.47, 128.57, 128.45, 122.77, $122.55,102.87,95.30,84.58,17.27$.

3-[(2-Methoxyphenyl)ethynyl]-5-methylpyridin-2-amine $12 b$. Using 2-amino-3-bromo-5-methylpyridine $(0.450 \quad \mathrm{~g}$, $2.41 \mathrm{mmol}), \quad$ 1-ethynyl-2-methoxybenzene $(0.3177 \mathrm{~g}$, $2.41 \mathrm{mmol})$, CuI (9.18 mg, $0.0482 \mathrm{mmol})$ and $\mathrm{Pd}\left(\mathrm{PPh}_{3}\right)_{4}$ (0.0557 g, $0.0482 \mathrm{mmol})$, the product 3-((2-methoxyphenyl)ethynyl)-5-methylpyridin-2-amine $\mathbf{1 2 b}(0.404 \mathrm{~g}$, 70\%) was obtained as a cream white solid. Mp: $120-123{ }^{\circ} \mathrm{C} ;{ }^{1} \mathrm{H}$ NMR $\left(500 \mathrm{MHz}, \mathrm{CDCl}_{3}\right) \delta 7.86(\mathrm{~s}, 1 \mathrm{H}), 7.49-7.39(\mathrm{~m}, 2 \mathrm{H}), 7.31$ (d, $J=$ 1.7, 1H), $6.94(\mathrm{ddd}, J=22.1,11.0,4.6,2 \mathrm{H}), 5.16(\mathrm{~s}, 2 \mathrm{H}), 3.92(\mathrm{~s}$, $3 \mathrm{H}), 2.18$ (s, 3H); ${ }^{13} \mathrm{C} \mathrm{NMR}\left(126 \mathrm{MHz}, \mathrm{CDCl}_{3}\right) \delta$ 159.90, 157.30, $147.73,139.55,132.45,129.87,122.17,120.62$, 112.19, 110.53, 103.13, 92.05, 89.43, 55.80, 17.29; IR ( $\left.\mathrm{cm}^{-1}\right) 3301$ (NH str.), 3001 (CH str.), 2212 (alkyne), $1639(\mathrm{C}=\mathrm{N}), 1594(\mathrm{C}=\mathrm{C}), 1179$ (C-O); HRMS $\left(\mathrm{ES}^{+}\right)$Calculated for $\mathrm{C}_{15} \mathrm{H}_{15} \mathrm{~N}_{2} \mathrm{O}[\mathrm{M}+\mathrm{H}]^{+}$: 239.1184, found 239.1198.

3-[(4-Methoxyphenyl)ethynyl]-5-methylpyridin-2-amine 12c. Using 2-amino-3-bromo-5-methylpyridine (0.450 g, $2.41 \mathrm{mmol})$, 1-ethynyl-4-methoxybenzene $(0.3177 \mathrm{~g}, 2.41 \mathrm{mmol}), \quad \mathrm{CuI}$ (9.18 $\mathrm{mg}, \quad 0.0482 \mathrm{mmol})$ and $\mathrm{Pd}\left(\mathrm{PPh}_{3}\right)_{4} \quad(0.0557 \mathrm{~g}$, $0.0482 \mathrm{mmol})$, the desired product 12c $(0.472 \mathrm{~g}, 82 \%)$ was obtained as a cream white solid. Mp: 94-96 ${ }^{\circ} \mathrm{C} ;{ }^{1} \mathrm{H}$ NMR $\left(500 \mathrm{MHz}, \mathrm{CDCl}_{3}\right) \delta 7.86(\mathrm{~d}, J=1.6,1 \mathrm{H}), 7.46-7.43(\mathrm{~m}, 2 \mathrm{H})$, $7.42(\mathrm{~d}, J=1.9,1 \mathrm{H}), 6.91-6.85(\mathrm{~m}, 2 \mathrm{H}), 4.92(\mathrm{~s}, 2 \mathrm{H}), 3.82(\mathrm{~s}$, $3 \mathrm{H}), 2.18$ (s, 3H); ${ }^{13} \mathrm{C}$ NMR (126 MHz, $\left.\mathrm{CDCl}_{3}\right) \delta$ 159.87, 156.86, $147.56,140.38,132.97,122.51,114.90,114.12$, 103.24, 95.30, 83.30, 55.33, 17.26; IR ( $\mathrm{cm}^{-1}$ ) 3276 (NH str.), 2987 (CH str.), 2238 (alkyne), $1618(\mathrm{C}=\mathrm{N}), 1564(\mathrm{C}=\mathrm{C}), 1170(\mathrm{C}-\mathrm{O})$; HRMS $\left(\mathrm{ES}^{+}\right.$) Calculated for $\mathrm{C}_{15} \mathrm{H}_{15} \mathrm{~N}_{2} \mathrm{O}[\mathrm{M}+\mathrm{H}]^{+}:$239.1184, found 239.1185 .

3-[(3-Chlorophenyl)ethynyl]-5-methylpyridin-2-amine 12d. Using 2-amino-3-bromo-5-methylpyridine (0.450 g, $2.41 \mathrm{mmol}$ ), 1-ethynyl-3-chlorobenzene $(0.328 \mathrm{~g}, 2.41 \mathrm{mmol})$, CuI $(9.18 \mathrm{mg}$, $0.0482 \mathrm{mmol})$ and $\mathrm{Pd}\left(\mathrm{PPh}_{3}\right)_{4}(0.0557 \mathrm{~g}, 0.0482 \mathrm{mmol})$, the product 12d (0.482 $\mathrm{g}, 83 \%)$ was obtained as a cream white solid. Mp: $104-106{ }^{\circ} \mathrm{C} ;{ }^{1} \mathrm{H}$ NMR $\left(500 \mathrm{MHz}, \mathrm{CDCl}_{3}\right) \delta 7.89$ (s, $1 \mathrm{H}), 7.50$ (s, 1H), 7.45 (s, 1H), 7.39 (d, $J=7.3,1 \mathrm{H}), 7.31(\mathrm{dt}, J=$ 13.3, 6.8, 2H), 4.93 (s, 2H), $2.20(\mathrm{~s}, 3 \mathrm{H}) ;{ }^{13} \mathrm{C} \mathrm{NMR}(126 \mathrm{MHz}$, $\left.\mathrm{CDCl}_{3}\right) \delta 156.84,148.16,140.86,134.35,131.31,129.70,129.58$, 128.84, 124.49, 122.68, 102.36, 93.87, 85.78, 17.25; IR $\left(\mathrm{cm}^{-1}\right)$ 3347 (NH str.), 3005 (CH str.), 2259 (alkyne), $1624(\mathrm{C}=\mathrm{N}), 1590$ $(\mathrm{C}=\mathrm{C}), 1176(\mathrm{C}-\mathrm{O})$; HRMS $\left(\mathrm{ES}^{+}\right)$Calculated for $\mathrm{C}_{14} \mathrm{H}_{12} \mathrm{~N}_{2} \mathrm{Cl}$ $[\mathrm{M}+\mathrm{H}]^{+}$: 243.0689, found 243.0705.

\section{General procedure for the Sonogashira reaction to synthesize} 13a-b and 14a-e

To a flame-dried round-bottom flask under a nitrogen or argon atmosphere containing 2-amino-3-iodopyridine derivative 9 and 10 was added $\mathrm{CuI}(2 \mathrm{~mol} \%)$ and $\operatorname{Pd}\left(\mathrm{PPh}_{3}\right)_{4}(2 \mathrm{~mol} \%)$ in one portion followed by the addition of the degassed alkyne solution in THF $(20 \mathrm{ml})$. Triethylamine $(5 \mathrm{ml})$ was then added and the reaction mixture was stirred at rt, until no starting material was present as monitored by thin layer chromatography (TLC). After this time, the reaction was quenched with a saturated aqueous ammonium chloride and was extracted with either dichloromethane or ethyl acetate. The combined organic extracts were dried over magnesium sulfate, filtered through either silica or celite and the excess solvent was removed on a rotary evaporator, followed by purification using flash chromatography (30\% EtOAc-hexane) to yield the desired products $12 \mathbf{a}-\mathbf{b}$ and $14 \mathbf{a}-\mathbf{e}$.

5-Chloro-3-[(trimethylsilyl)ethynyl $]$ pyridin-2-amine 13a. Using 2-amino-3-iodo-5-chloropyridine (4.475 g, $17.6 \mathrm{mmol}$ ), trimethylsilylacetylene (8.626 g, $88.1 \mathrm{mmol}), \mathrm{CuI}(0.06704 \mathrm{~g}$, $0.352 \mathrm{mmol})$ and $\mathrm{Pd}\left(\mathrm{PPh}_{3}\right)_{4}(0.4068 \mathrm{~g}, 0.352 \mathrm{mmol})$ 13a $(3.626 \mathrm{~g}, 92 \%)$ was obtained as a cream white solid ${ }^{17}(0.2153 \mathrm{~g}$, $92 \%) .{ }^{1} \mathrm{H}$ NMR (300 MHz, $\left.\mathrm{CDCl}_{3}\right) \delta 7.96(\mathrm{~d}, J=2.4,1 \mathrm{H}), 7.51$ $(\mathrm{d}, J=2.5,1 \mathrm{H}), 5.06(\mathrm{~s}, 2 \mathrm{H}), 0.27(\mathrm{~s}, 9 \mathrm{H})$.

5-Chloro-3-[(4-methoxy-2-methylphenyl)ethynyl]pyridin-2-amine 13b. Using 2-amino-3-iodo-5-chloropyridine $(0.300 \mathrm{~g}$, $1.19 \mathrm{mmol})$, 1-ethynyl-4-methoxy-2-methylbenzene $(0.1740 \mathrm{~g}$, $1.19 \mathrm{mmol})$, CuI (4.517 $\mathrm{mg}, 0.0237 \mathrm{mmol})$ and $\mathrm{Pd}\left(\mathrm{PPh}_{3}\right)_{4}$ $(0.4068 \mathrm{~g}, 0.0237 \mathrm{mmol})$ the product $13 \mathbf{b}$ was produced $(0.2834 \mathrm{~g}, 87 \%)$ as a cream white solid. Mp: $127-130{ }^{\circ} \mathrm{C}$; 
${ }^{1} \mathrm{H}$ NMR (500 MHz, $\left.\mathrm{CDCl}_{3}\right) \delta 7.97(\mathrm{~s}, 1 \mathrm{H}), 7.55(\mathrm{~s}, 1 \mathrm{H}), 7.41(\mathrm{~d}$, $J=8.4,1 \mathrm{H}), 6.79(\mathrm{~s}, 1 \mathrm{H}), 6.74(\mathrm{~d}, J=8.4,1 \mathrm{H}), 5.04(\mathrm{~s}, 2 \mathrm{H}), 3.83$ $(\mathrm{s}, 3 \mathrm{H}), 2.48(\mathrm{~s}, 3 \mathrm{H}) ;{ }^{13} \mathrm{C} \mathrm{NMR}\left(126 \mathrm{MHz}, \mathrm{CDCl}_{3}\right) \delta 160.17$, 156.92, 145.88, 141.88, 138.66, 133.38, 120.18, 115.32, 114.26, 111.56, 105.04, 95.67, 85.77, 55.31, 21.18; IR $\left(\mathrm{cm}^{-1}\right) 3368(\mathrm{NH}$ str.), 2989 (CH str.), 2287 (alkyne), $1605(\mathrm{C}=\mathrm{N}), 1558(\mathrm{C}=\mathrm{C})$, 1164 (C-O); HRMS (ES ${ }^{+}$) Calculated for $\mathrm{C}_{15} \mathrm{H}_{14} \mathrm{~N}_{2} \mathrm{ClO}[\mathrm{M}+\mathrm{H}]^{+}$: 273.0795, found 273.0801 .

3-(2-Amino-5-bromopyridin-3-yl)prop-2-yn-1-yl benzoate $14 a$. Using 2-amino-3-iodo-5-bromopyridine (2.600 g, $8.70 \mathrm{mmol})$, prop-2-yn-1-yl benzoate (1.672 g, $16.4 \mathrm{mmol})$, CuI (33.14 mg, $0.174 \mathrm{mmol})$ and $\mathrm{Pd}\left(\mathrm{PPh}_{3}\right)_{4}(0.2011 \mathrm{~g}, 0.174 \mathrm{mmol})$ 14a $(2.546 \mathrm{~g}, 88 \%)$ was obtained as a brown solid. Mp: $126-128{ }^{\circ} \mathrm{C}$; ${ }^{1} \mathrm{H}$ NMR (300 MHz, $\left.\mathrm{CDCl}_{3}\right) \delta 8.11-8.02(\mathrm{~m}, 3 \mathrm{H}), 7.63-7.53(\mathrm{~m}$, $2 \mathrm{H}), 7.45(\mathrm{t}, J=7.6,2 \mathrm{H}), 5.23(\mathrm{~s}, 2 \mathrm{H}), 5.14(\mathrm{~s}, 2 \mathrm{H}) ;{ }^{13} \mathrm{C} \mathrm{NMR}$ $\left(75 \mathrm{MHz}, \mathrm{CDCl}_{3}\right){ }^{13} \mathrm{C} \mathrm{NMR}\left(75 \mathrm{MHz}, \mathrm{CDCl}_{3}\right) \delta$ 166.30, 158.39, 149.54, 142.34, 133.77, 130.12, 129.63, 128.80, 106.91, 103.77, 90.95, 81.23, 53.41; IR ( $\mathrm{cm}^{-1}$ ) 3249 (NH str.), 2989 (CH str.), 2312 (alkyne), $1629(\mathrm{C}=\mathrm{N}), 1548(\mathrm{C}=\mathrm{C}), 1173(\mathrm{C}-\mathrm{O})$; HRMS $\left(\mathrm{ES}^{+}\right)$Calculated for $\mathrm{C}_{15} \mathrm{H}_{12} \mathrm{~N}_{2} \mathrm{O}_{2} \mathrm{Br}[\mathrm{M}+\mathrm{H}]^{+}$: 331.0082, found 331.0080 .

5-Bromo-3-(3-morpholinoprop-1-yn-1-yl)pyridin-2-amine $14 \mathrm{~b}$. Starting with 2-amino-3-iodo-5-bromopyridine $(3.000 \mathrm{~g}$, $10.0 \mathrm{mmol}), \quad 4-($ prop-2-yn-1-yl)morpholine $(1.253 \mathrm{~g}$, $10.0 \mathrm{mmol})$, CuI (38.23 $\mathrm{mg}, 0.201 \mathrm{mmol})$ and $\mathrm{Pd}\left(\mathrm{PPh}_{3}\right)_{4}$ (0.2320 g, $0.201 \mathrm{mmol})$, the product 5-bromo-3-(3-morpholinoprop-1-yn-1-yl)pyridin-2-amine 14b (2.541 g, 86\%) was obtained as a brown solid. Mp: $115-118{ }^{\circ} \mathrm{C} ;{ }^{1} \mathrm{H} \quad \mathrm{NMR}$ $\left(300 \mathrm{MHz}, \mathrm{CDCl}_{3}\right) \delta 8.01(\mathrm{~d}, J=2.4,1 \mathrm{H}), 7.57(\mathrm{~d}, J=2.4,1 \mathrm{H})$, 5.14 (s, 2H), 3.80-3.66 (m, 4H), 3.53 (s, 2H), 2.66-2.50 (m, 4H); ${ }^{13} \mathrm{C}$ NMR (75 $\left.\mathrm{MHz}, \mathrm{CDCl}_{3}\right) \delta 157.94,148.74,142.22,107.06$, 104.78, 91.93, 80.05, 67.05, 52.68, 48.38; IR $\left(\mathrm{cm}^{-1}\right) 3310(\mathrm{NH}$ str.), 2959 (CH str.), 2291 (alkyne), $1630(\mathrm{C}=\mathrm{N}), 1572(\mathrm{C}=\mathrm{C})$, $1132(\mathrm{C}-\mathrm{O})$; HRMS $\left(\mathrm{ES}^{+}\right)$Calculated for $\mathrm{C}_{12} \mathrm{H}_{15} \mathrm{~N}_{3} \mathrm{BrO}[\mathrm{M}+\mathrm{H}]^{+}$: 296.0398, found 296.0420 .

5-Bromo-3-[3-( piperidin-1-yl)prop-1-yn-1-yl]pyridin-2-amine 14c. Using 2-amino-3-iodo-5-bromopyridine $(3.000 \mathrm{~g}$, $10.0 \mathrm{mmol}$ ), 1-(prop-2-yn-1-yl)piperidine (1.235 g, $10.0 \mathrm{mmol}$ ), CuI (38.23 mg, $0.201 \mathrm{mmol})$ and $\mathrm{Pd}\left(\mathrm{PPh}_{3}\right)_{4}(0.2320 \mathrm{~g}$, $0.201 \mathrm{mmol})$ the product $14 \mathrm{c}(2.451 \mathrm{~g}, 83 \%)$ was obtained as brown solid. Mp: $79-81{ }^{\circ} \mathrm{C} ;{ }^{1} \mathrm{H}$ NMR $\left(300 \mathrm{MHz}, \mathrm{CDCl}_{3}\right) \delta 7.99$ $(\mathrm{d}, J=2.4,1 \mathrm{H}), 7.57(\mathrm{~d}, J=2.4,1 \mathrm{H}), 5.15(\mathrm{~s}, 2 \mathrm{H}), 3.50(\mathrm{~s}, 2 \mathrm{H})$, $2.52(\mathrm{~m}, 4 \mathrm{H}), 1.61$ (dt, $J=11.0,5.6,4 \mathrm{H}), 1.42(\mathrm{~d}, J=5.1,2 \mathrm{H})$; ${ }^{13} \mathrm{C}$ NMR (75 $\left.\mathrm{MHz}, \mathrm{CDCl}_{3}\right) \delta 157.99,148.50,142.11,107.04$, 105.15, 92.92, 79.53, 53.75, 48.81, 26.16, 24.09; IR (cm $\left.{ }^{-1}\right) 3262$ (NH str.), 2988 (CH str.), 2259 (alkyne), $1639(\mathrm{C}=\mathrm{N}), 1558$ $(\mathrm{C}=\mathrm{C})$; HRMS $\left(\mathrm{ES}^{+}\right)$Calculated for $\mathrm{C}_{13} \mathrm{H}_{17} \mathrm{~N}_{3} \mathrm{Br}[\mathrm{M}+\mathrm{H}]^{+}$: 294.0606, found 294.0613.

3-[(3,5-Bis(trifluoromethyl)phenyl)ethynyl]-5-bromopyridin2-amine 14d. Using 2-amino-3-iodo-5-bromopyridine (1.500 g, $5.02 \mathrm{mmol}$ ), 1-ethynyl-3,5(trifluoromethyl)benzene (1.235 g, $10.0 \mathrm{mmol})$, CuI (19.12 $\mathrm{mg}, 0.100 \mathrm{mmol})$ and $\mathrm{Pd}\left(\mathrm{PPh}_{3}\right)_{4}$ $(0.1160 \mathrm{~g}, 0.100 \mathrm{mmol})$ the product $14 \mathrm{~d}(1.436 \mathrm{~g}, 70 \%)$ was obtained as white crystals. Mp: $194-196{ }^{\circ} \mathrm{C} ;{ }^{1} \mathrm{H}$ NMR $\left(500 \mathrm{MHz} \mathrm{CDCl}_{3}\right) \delta 8.14(\mathrm{~d}, J=2.4,1 \mathrm{H}), 7.94(\mathrm{~s}, 2 \mathrm{H}), 7.86(\mathrm{~s}$, $1 \mathrm{H}), 7.74(\mathrm{~d}, J=2.4,1 \mathrm{H}), 5.10(\mathrm{~s}, 2 \mathrm{H}) ;{ }^{13} \mathrm{C}$ NMR $(126 \mathrm{MHz}$,
$\left.\mathrm{CDCl}_{3}\right) \delta 157.71,150.16,142.56,132.76,132.49,131.74,131.72$, 124.92, 124.25, 122.64, 122.08, 107.59, 103.59, 93.49, 86.93; IR $\left(\mathrm{cm}^{-1}\right) 3309$ (NH str.), 2995 (CH str.), 2319 (alkyne), 1629 $(\mathrm{C}=\mathrm{N}), 1571(\mathrm{C}=\mathrm{C})$; HRMS $\left(\mathrm{ES}^{+}\right)$Calculated for $\mathrm{C}_{15} \mathrm{H}_{8} \mathrm{~N}_{2} \mathrm{BrF}_{6}$ $[\mathrm{M}+\mathrm{H}]^{+}:$408.9775, found 408.9778.

5-Bromo-3-[(2-methoxyphenyl)ethynyl]pyridin-2-amine $14 \mathrm{e}$. Using 2-amino-3-iodo-5-bromopyridine (2.500 g, $8.36 \mathrm{mmol}$ ), 1-ethynyl-2-methoxybenzene $(1.105 \mathrm{~g}, 8.36 \mathrm{mmol}), \quad \mathrm{CuI}$ (31.84 mg, $0.167 \mathrm{mmol}), \mathrm{Pd}\left(\mathrm{PPh}_{3}\right)_{4}(0.1932 \mathrm{~g}, 0.167 \mathrm{mmol})$ furnished the product 14e as a cream white solid $(2.013 \mathrm{~g}$, 83\%). Mp: $126-128{ }^{\circ} \mathrm{C} ;{ }^{1} \mathrm{H}$ NMR (300 MHz, $\left.\mathrm{CDCl}_{3}\right) \delta 8.06$ (d, $J=2.3,1 \mathrm{H}), 7.67(\mathrm{~d}, J=2.3,1 \mathrm{H}), 7.46(\mathrm{dd}, J=7.5,1.5,1 \mathrm{H})$, 7.40-7.29 (m, 1H), 7.00-6.91 (m, 2H), 5.51 (s, 2H), $3.92(\mathrm{~s}, 3 \mathrm{H})$; ${ }^{13} \mathrm{C}$ NMR (75 $\left.\mathrm{MHz}, \mathrm{CDCl}_{3}\right) \delta 160.00,157.82,148.21,140.39$, $132.47,130.34,120.70,111.56,110.55,106.69$, 105.26, 93.51, 88.04, 55.81; IR ( $\mathrm{cm}^{-1}$ ) 3310 (NH str.), 3002 (CH str.), 2358 (alkyne), $1627(\mathrm{C}=\mathrm{N}), 1571(\mathrm{C}=\mathrm{C}), 1181(\mathrm{C}-\mathrm{O})$; HRMS $\left(\mathrm{ES}^{+}\right)$Calculated for $\mathrm{C}_{14} \mathrm{H}_{12} \mathrm{~N}_{2} \mathrm{BrO}[\mathrm{M}+\mathrm{H}]^{+}$: 303.0133, found 303.0147.

General method for the synthesis of azaindoles 15a-c, 16a-d, 17a-b and 18a-e

The corresponding 2-amino-3-ethynylpyridine derivatives (40-200 mg) 11a-c, 12a-d, 13a-b and 14a-e were dissolved in MeCN. To the resulting solution was added trifluoroacetic acid ( 1 eq.) in one portion followed by the addition of trifluoroacetic anhydride (1.3 eq.) dropwise. The resulting mixture was then heated to reflux for $8 \mathrm{~h}$ and was allowed to cool to rt. The solvent was then removed on a rotary evaporator and the resulting crude material was re-dissolved in dichloromethane and washed with aqueous sodium carbonate (10\%). The separated and dried $\left(\mathrm{MgSO}_{4}\right)$ organic phase was purified by flash silica chromatography using ethyl acetate-hexane mixture (10-50\%) gave desired azaindoles 15a-c, 16a-d, 17a-d and 18a-e in good yields.

2-(2-Methoxyphenyl)-1H-pyrrolo[2,3-b]pyridine 15a. The product 15a was produced as a cream white solid $(0.1236 \mathrm{~g}$, 92\%). Mp: $125-128{ }^{\circ} \mathrm{C} ;{ }^{1} \mathrm{H}$ NMR (500 MHz, $\mathrm{CDCl}_{3}$ ) $\delta 10.47$ (s, $1 \mathrm{H}), 8.30-8.20(\mathrm{~m}, 1 \mathrm{H}), 7.93-7.87(\mathrm{~m}, 1 \mathrm{H}), 7.84(\mathrm{dd}, J=7.7$, 1.5, 1H), 7.36-7.27 (m, 1H), 7.12-6.98 (m, 3H), 6.84 (d, $J=1.6$, $1 \mathrm{H}), 3.98(\mathrm{~s}, 3 \mathrm{H}) ;{ }^{13} \mathrm{C} \mathrm{NMR}\left(126 \mathrm{MHz}, \mathrm{CDCl}_{3}\right) \delta$ 156.08, 148.60, 142.91, 136.27, 129.31, 128.23, 128.04, 121.46, 120.66, 120.05, 116.14, 111.91, 98.09, 55.76; IR ( $\left.\mathrm{cm}^{-1}\right) 3234$ (NH str.), 2933 (CH str.), $1626(\mathrm{C}=\mathrm{N}), 1578(\mathrm{C}=\mathrm{C}), 1179(\mathrm{C}-\mathrm{O})$; HRMS $\left(\mathrm{ES}^{+}\right)$ Calculated for $\mathrm{C}_{14} \mathrm{H}_{13} \mathrm{~N}_{2} \mathrm{O}[\mathrm{M}+\mathrm{H}]^{+}$: 225.1028, found 225.1042.

2-(4-Methoxyphenyl)-1H-pyrrolo[2,3-b]pyridine 15b. The product 15b was furnished as a cream white solid $(0.1542 \mathrm{~g}$, 88\%). Mp: $182-184{ }^{\circ} \mathrm{C} ;{ }^{1} \mathrm{H}$ NMR (500 MHz, $\left.\mathrm{CDCl}_{3}\right) \delta 12.35$ (s, $1 \mathrm{H}), 8.25(\mathrm{~d}, J=3.3,1 \mathrm{H}), 7.92(\mathrm{~d}, J=7.7,1 \mathrm{H}), 7.82(\mathrm{~d}, J=8.8$, 2H), 7.15-6.97 (m, 3H), 6.67 (s, 1H), $3.90(\mathrm{~s}, 3 \mathrm{H}) ;{ }^{13} \mathrm{C} \mathrm{NMR}$ $\left(126 \mathrm{MHz}, \mathrm{CDCl}_{3}\right) \delta 159.83,149.92,141.64,139.57,128.25$, $127.21,125.15,122.56,116.06,114.52,96.24,55.44 ;$ IR $\left(\mathrm{cm}^{-1}\right)$ 3281 (NH str.), 2986 (CH str.), $1671(\mathrm{C}=\mathrm{N}), 1594(\mathrm{C}=\mathrm{C}), 1176$ (C-O); HRMS $\left(\mathrm{ES}^{+}\right)$Calculated for $\mathrm{C}_{14} \mathrm{H}_{13} \mathrm{~N}_{2} \mathrm{O}[\mathrm{M}+\mathrm{H}]^{+}$: 225.1028 , found 225.1035 .

2-(3-Chlorophenyl)-1H-pyrrolo[2,3-b]pyridine 15c. The azaindole 15c was produced as a cream white solid $0.08213 \mathrm{~g}$, 
81\%). Mp: $205-207{ }^{\circ} \mathrm{C} ;{ }^{1} \mathrm{H}$ NMR (500 MHz, $\left.\mathrm{CDCl}_{3}\right) \delta 11.82(\mathrm{~s}$, $1 \mathrm{H}), 8.37(\mathrm{~s}, 1 \mathrm{H}), 7.98(\mathrm{~d}, J=7.9,1 \mathrm{H}), 7.86(\mathrm{~s}, 1 \mathrm{H}), 7.72(\mathrm{~d}, J=$ $7.5,1 \mathrm{H}), 7.44(\mathrm{t}, J=7.9,1 \mathrm{H}), 7.37$ (d, $J=7.8,1 \mathrm{H}), 7.14(\mathrm{~s}, 1 \mathrm{H})$, $6.82(\mathrm{~s}, 1 \mathrm{H}) ;{ }^{13} \mathrm{C} \mathrm{NMR}\left(126 \mathrm{MHz}, \mathrm{CDCl}_{3}\right) \delta 149.76,143.05$, 137.60, 135.14, 134.05, 130.36, 129.12, 128.18, 125.73, 123.79, 122.03, 116.56, 98.53; IR ( $\left.\mathrm{cm}^{-1}\right) 3255$ (NH str.), 2987 (CH str.), $1631(\mathrm{C}=\mathrm{N}), 1588(\mathrm{C}=\mathrm{C})$; HRMS $\left(\mathrm{ES}^{+}\right)$Calculated for $\mathrm{C}_{13} \mathrm{H}_{10} \mathrm{~N}_{2} \mathrm{Cl}[\mathrm{M}+\mathrm{H}]^{+}$: 229.0533, found 229.0536.

5-Methyl-2-phenyl-1H-pyrrolo[2,3-b]pyridine 16a. The product 16a was formed as a cream white $\operatorname{solid}^{15}(86 \%) .{ }^{1} \mathrm{H}$ NMR (500 MHz, DMSO) $\delta 11.98(\mathrm{~s}, 1 \mathrm{H}), 8.07$ (d, $J=1.3,1 \mathrm{H})$, $7.93(\mathrm{~d}, J=7.5,2 \mathrm{H}), 7.72(\mathrm{~s}, 1 \mathrm{H}), 7.46(\mathrm{t}, J=7.7,2 \mathrm{H}), 7.34(\mathrm{t}, J=$ $7.4,1 \mathrm{H}), 6.84(\mathrm{~d}, J=1.8,1 \mathrm{H}), 2.37(\mathrm{~s}, 3 \mathrm{H}) ;{ }^{13} \mathrm{C}$ NMR $(126 \mathrm{MHz}$, DMSO) $\delta 148.33,143.62,138.25,131.71,128.84,127.83$, 127.56, 125.19, 124.37, 120.73, 96.49, 18.09 .

2-(2-Methoxyphenyl)-5-methyl-1H-pyrrolo[2,3-b]pyridine $16 \mathrm{~b}$. The desired azaindole 16b was furnished as a cream white solid (0.1001 g, 94\%). Mp: $169-171{ }^{\circ} \mathrm{C} ;{ }^{1} \mathrm{H}$ NMR $(500 \mathrm{MHz}$, $\left.\mathrm{CDCl}_{3}\right) \delta 10.21(\mathrm{~s}, 1 \mathrm{H}), 8.10(\mathrm{~s}, 1 \mathrm{H}), 7.83(\mathrm{dd}, J=7.8,1.6,1 \mathrm{H})$, 7.69 (s, 1H), 7.33-7.26 (m, 1H), 7.05 (ddd, $J=13.7,10.3,4.6$, $2 \mathrm{H}), 6.75(\mathrm{~d}, J=2.0,1 \mathrm{H}), 3.98(\mathrm{~s}, 3 \mathrm{H}), 2.42(\mathrm{~s}, 3 \mathrm{H}) ;{ }^{13} \mathrm{C} \mathrm{NMR}$ $\left(126 \mathrm{MHz}, \mathrm{CDCl}_{3}\right) \delta 155.99,147.26,143.95,136.31,129.14$, 128.10, 127.97, 125.04, 121.45, 120.41, 120.14, 111.91, 97.37, 55.75, 18.60; IR ( $\mathrm{cm}^{-1}$ ) 3240 (NH str.), 2981 (CH str.), 1624 $(\mathrm{C}=\mathrm{N}), 1579(\mathrm{C}=\mathrm{C}), 1178(\mathrm{C}-\mathrm{O})$; HRMS $\left(\mathrm{ES}^{+}\right)$Calculated for $\mathrm{C}_{15} \mathrm{H}_{15} \mathrm{~N}_{2} \mathrm{O}[\mathrm{M}+\mathrm{H}]^{+}$: 239.1184, found 269.1190.

2-(4-Methoxyphenyl)-5-methyl-1H-pyrrolo[2,3-b]pyridine 16c. The product $\mathbf{1 6 c}$ was produced as a cream white solid (0.1011 g, 85\%). Mp: 245-247 ${ }^{\circ} \mathrm{C} ;{ }^{1} \mathrm{H}$ NMR (500 MHz, MeOD) $\delta 7.99(\mathrm{~s}, 1 \mathrm{H}), 7.78(\mathrm{~d}, J=8.8,2 \mathrm{H}), 7.75(\mathrm{~s}, 1 \mathrm{H}), 7.04(\mathrm{~d}, J=8.8$, 2H), 6.64 (s, 1H), 3.87 (s, 3H), 2.43 (s, 3H); ${ }^{13} \mathrm{C}$ NMR (126 MHz, MeOD) $\delta 161.32,149.06,143.26,140.97,129.54,129.51$, 127.96, 126.28, 126.05, 115.41, 96.61, 55.83, 18.50; IR $\left(\mathrm{cm}^{-1}\right)$ 3298 (NH str.), 2972 (CH str.), $1635(\mathrm{C}=\mathrm{N}), 1575(\mathrm{C}=\mathrm{C}), 1168$ (C-O); HRMS $\left(\mathrm{ES}^{+}\right)$Calculated for $\mathrm{C}_{15} \mathrm{H}_{15} \mathrm{~N}_{2} \mathrm{O}[\mathrm{M}+\mathrm{H}]^{+}$: 239.1184, found 239.1190.

2-(3-Chlorophenyl)-5-methyl-1H-pyrrolo[2,3-b]pyridine $\mathbf{1 6 d}$. The product 16d was formed as a cream white solid $(0.1012 \mathrm{~g}$, 82\%). Mp: 239-242 ${ }^{\circ} \mathrm{C} ;{ }^{1} \mathrm{H}$ NMR (500 MHz, DMSO) $\delta 12.07$ (s, $1 \mathrm{H}), 8.10(\mathrm{~s}, 1 \mathrm{H}), 8.03(\mathrm{~s}, 1 \mathrm{H}), 7.90(\mathrm{~d}, J=7.7,1 \mathrm{H}), 7.75(\mathrm{~s}, 1 \mathrm{H})$, $7.48(\mathrm{t}, J=7.6,1 \mathrm{H}), 7.39(\mathrm{~d}, J=7.8,1 \mathrm{H}), 6.97(\mathrm{~s}, 1 \mathrm{H}), 2.38(\mathrm{~s}$, $3 \mathrm{H}) ;{ }^{13} \mathrm{C}$ NMR (126 MHz, DMSO) $\delta$ 148.34, 144.28, 136.55, 133.85, 133.80, 130.69, 127.90, 127.46, 124.75, 124.63, 123.76, 120.52, 97.78, 18.07; IR ( $\mathrm{cm}^{-1}$ ) 3347 (NH str.), 2947 (CH str.), $1630(\mathrm{C}=\mathrm{N}), 1587(\mathrm{C}=\mathrm{C})$; HRMS $\left(\mathrm{ES}^{+}\right)$Calculated for $\mathrm{C}_{14} \mathrm{H}_{12} \mathrm{~N}_{2} \mathrm{Cl}[\mathrm{M}+\mathrm{H}]^{+}:$243.0689, found 243.0697.

5-Chloro-1H-pyrrolo[2,3-b]pyridine 17a. The azaindole 17a was yielded as a cream white solid ${ }^{16}(0.1032 \mathrm{~g}, 84 \%) .{ }^{1} \mathrm{H}$ NMR $\left(300 \mathrm{MHz}, \mathrm{CDCl}_{3}\right) \delta 10.89(\mathrm{~s}, 1 \mathrm{H}), 8.29(\mathrm{~d}, J=2.0,1 \mathrm{H}), 7.94(\mathrm{~d}$, $J=2.0,1 \mathrm{H}), 7.46-7.35(\mathrm{~m}, 1 \mathrm{H}), 6.62-6.24(\mathrm{~m}, 1 \mathrm{H}) ;{ }^{13} \mathrm{C} \mathrm{NMR}$ $\left(75 \mathrm{MHz}, \mathrm{CDCl}_{3}\right) \delta 146.94,141.20,128.27,126.86,123.80$, 121.28, 100.59; IR ( $\mathrm{cm}^{-1}$ ) 3288 (NH str.), 2969 (CH str.), 1629 $(\mathrm{C}=\mathrm{N}), 1553(\mathrm{C}=\mathrm{C})$.

5-Chloro-2-(4-methoxy-2-methylphenyl)-1H-pyrrolo[2,3-b]pyridine $\mathbf{1 7 b}$. The desired product $\mathbf{1 7} \mathbf{b}$ was produced as a yellow solid (0.08109 g, 80\%). Mp: 209-211 ${ }^{\circ} \mathrm{C} ;{ }^{1} \mathrm{H}$ NMR $(500 \mathrm{MHz}$,
$\left.\mathrm{CDCl}_{3}\right) \delta 10.13(\mathrm{~s}, 1 \mathrm{H}), 8.10(\mathrm{~s}, 1 \mathrm{H}), 7.88(\mathrm{~s}, 1 \mathrm{H}), 7.47(\mathrm{~d}, J=8.2$, $1 \mathrm{H}), 6.89(\mathrm{~s}, 2 \mathrm{H}), 6.44(\mathrm{~s}, 1 \mathrm{H}), 3.88(\mathrm{~s}, 3 \mathrm{H}), 2.50(\mathrm{~s}, 3 \mathrm{H}) ;{ }^{13} \mathrm{C}$ NMR (126 MHz, $\left.\mathrm{CDCl}_{3}\right) \delta 159.87,147.00,140.74,140.15$, 137.90, 130.45, 127.34, 124.29, 123.95, 122.37, 116.68, 111.72, 99.84, 55.36, 21.28; IR ( $\left.\mathrm{cm}^{-1}\right) 3210$ (NH str.), 2989 (CH str.), $1637(\mathrm{C}=\mathrm{N}), 1571(\mathrm{C}=\mathrm{C}), 1182(\mathrm{C}-\mathrm{O}) . \mathrm{HRMS}\left(\mathrm{ES}^{+}\right)$Calculated for $\mathrm{C}_{15} \mathrm{H}_{14} \mathrm{~N}_{2} \mathrm{ClO}[\mathrm{M}+\mathrm{H}]^{+}$: 273.0795, found 273.0803.

(5-Bromo-1H-pyrrolo[2,3-b]pyridin-2-yl)methyl benzoate $18 \mathrm{a}$. The azaindole 18a was formed as a cream white solid (0.4211 g, 83\%). Mp: 181-184 ${ }^{\circ} \mathrm{C} ;{ }^{1} \mathrm{H}$ NMR (500 MHz, MeOD) $\delta 8.33(\mathrm{~s}, 1 \mathrm{H}), 8.27(\mathrm{~d}, J=2.2,1 \mathrm{H}), 8.15(\mathrm{~d}, J=2.2,1 \mathrm{H})$, 8.10-8.07 (m, 2H), $7.64(\mathrm{t}, J=7.5,1 \mathrm{H}), 7.50(\mathrm{t}, J=7.8,2 \mathrm{H}), 6.60$ $(\mathrm{s}, 1 \mathrm{H}), 5.52(\mathrm{~s}, 2 \mathrm{H}) ;{ }^{13} \mathrm{C}$ NMR $(126 \mathrm{MHz}, \mathrm{MeOD}) \delta$ 167.67, 148.54, 144.41, 137.76, 134.49, 132.25, 131.11, 130.77, 130.71, 129.68, 123.83, 112.41, 101.66, 60.78; IR ( $\left.\mathrm{cm}^{-1}\right) 3368$ (NH str.), 3000 (CH str.), $1605(\mathrm{C}=\mathrm{N}), 1559(\mathrm{C}=\mathrm{C}), 1164(\mathrm{C}-\mathrm{O})$; HRMS $\left(\mathrm{ES}^{+}\right)$Calculated for $\mathrm{C}_{15} \mathrm{H}_{12} \mathrm{~N}_{2} \mathrm{BrO}_{2}[\mathrm{M}+\mathrm{H}]^{+}$: 331.0082, found 331.0100 .

4-[(5-Bromo-1H-pyrrolo[2,3-b]pyridin-2-yl)methyl]morpholine 18b. The product $18 \mathrm{~b}$ formed as a cream white solid $(0.159 \mathrm{~g}$, 79\%). Mp: $189-192{ }^{\circ} \mathrm{C} ;{ }^{1} \mathrm{H}$ NMR (300 MHz, $\left.\mathrm{CDCl}_{3}\right) \delta 11.21(\mathrm{~s}$, $1 \mathrm{H}), 8.37$ (d, $J=2.1,1 \mathrm{H}), 7.97(\mathrm{~d}, J=2.0,1 \mathrm{H}), 6.28(\mathrm{~s}, 1 \mathrm{H})$, 3.78-3.70 (m, 6H), 2.59-2.44 (m, 4H); ${ }^{13} \mathrm{C}$ NMR $(75 \mathrm{MHz}$, $\left.\mathrm{CDCl}_{3}\right) \delta 147.70,142.96,138.67,130.56,123.04,111.75$, 99.65, 67.19, 56.72, 54.07; IR ( $\mathrm{cm}^{-1}$ ) 3223 (NH str.), 2969 (CH str.), $1640(\mathrm{C}=\mathrm{N}), 1573(\mathrm{C}=\mathrm{C}), 1140(\mathrm{C}-\mathrm{O})$; HRMS $\left(\mathrm{ES}^{+}\right)$Calculated for $\mathrm{C}_{12} \mathrm{H}_{15} \mathrm{~N}_{3} \mathrm{BrO}[\mathrm{M}+\mathrm{H}]^{+}$: 296.0398, found 296.0401.

5-Bromo-2-(piperidin-1-ylmethyl)-1 $\mathrm{H}$-pyrrolo[2,3-b]pyridine 18c. The azaindole 18c was furnished as a cream white solid (0.1131 g, 83\%). Mp: 194-195 ${ }^{\circ} \mathrm{C} ;{ }^{1} \mathrm{H}$ NMR (300 MHz, $\mathrm{CDCl}_{3}$ ) $\delta 10.55(\mathrm{~s}, 1 \mathrm{H}), 8.35(\mathrm{~d}, J=2.1,1 \mathrm{H}), 7.94(\mathrm{~d}, J=2.0,1 \mathrm{H}), 6.23$ (s, 1H), 3.65 (s, 2H), 2.43-2.38 (m, 4H), 1.66-1.53 (m, 4H), 1.46 $(\mathrm{d}, J=4.9,2 \mathrm{H}) ;{ }^{13} \mathrm{C} \mathrm{NMR}\left(75 \mathrm{MHz}, \mathrm{CDCl}_{3}\right) \delta 147.50,142.89$, $139.93,130.25,123.12,111.74,98.93,57.02,55.11,26.28$, 24.55; IR ( $\left.\mathrm{cm}^{-1}\right) 3217$ (NH str.), 2989 (CH str.), $1631(\mathrm{C}=\mathrm{N})$, $1572(\mathrm{C}=\mathrm{C})$; HRMS $\left(\mathrm{ES}^{+}\right)$Calculated for $\mathrm{C}_{13} \mathrm{H}_{13} \mathrm{~N}_{3} \mathrm{Br}[\mathrm{M}+\mathrm{H}]^{+}$: 294.0606, found 294.0621.

2-[3,5-Bis(trifluoromethyl)phenyl]-5-bromo-1 $H$-pyrrolo[2,3-b]pyridine 18d. The azaindole $\mathbf{1 8 d}$ was produced as a white solid (0.1009 g, 78\%). Mp: 251-253 ${ }^{\circ} \mathrm{C} ;{ }^{1} \mathrm{H}$ NMR $(500 \mathrm{MHz}$, acetone) $\delta 11.69(\mathrm{~s}, 1 \mathrm{H}), 8.60(\mathrm{~s}, 2 \mathrm{H}), 8.33(\mathrm{~d}, J=2.2,1 \mathrm{H}), 8.21$ $(\mathrm{d}, J=2.2,1 \mathrm{H}), 8.03(\mathrm{~s}, 1 \mathrm{H}), 7.30(\mathrm{~s}, 1 \mathrm{H}), 6.43(\mathrm{~s}, 1 \mathrm{H}) ;{ }^{13} \mathrm{C} \mathrm{NMR}$ (126 MHz, acetone) $\delta$ 149.39, 145.63, 137.75, 134.97, 132.78, $131.55,126.75,126.72,125.47,123.56,123.30,122.19,112.87$, 100.82; IR ( $\left.\mathrm{cm}^{-1}\right) 3293$ (NH str.), 2952 ( $\mathrm{CH}$ str.), $1641(\mathrm{C}=\mathrm{N})$, $1576(\mathrm{C}=\mathrm{C})$; HRMS $\left(\mathrm{ES}^{+}\right)$Calculated for $\mathrm{C}_{15} \mathrm{H}_{8} \mathrm{~N}_{2} \mathrm{BrF}_{6}[\mathrm{M}+\mathrm{H}]^{+}$: 408.9775, found 408.9764 .

5-Bromo-2-(2-methoxyphenyl)-1H-pyrrolo[2,3-b]pyridine $18 \mathrm{e}$. The desired product 18e was produced as a cream white solid (0.1069, 80\%). Mp: 196-197 ${ }^{\circ} \mathrm{C} ;{ }^{1} \mathrm{H}$ NMR (300 MHz, $\mathrm{CDCl}_{3}$ ) $\delta 10.38(\mathrm{~s}, 1 \mathrm{H}), 8.30(\mathrm{~d}, J=1.7,1 \mathrm{H}), 8.02(\mathrm{~d}, J=1.6,1 \mathrm{H}), 7.84$ (dd, $J=7.8,1.6,1 \mathrm{H}), 7.43-7.32(\mathrm{~m}, 1 \mathrm{H}), 7.17-7.02(\mathrm{~m}, 2 \mathrm{H})$, $6.78(\mathrm{~d}, J=2.1,1 \mathrm{H}), 4.02(\mathrm{~s}, 3 \mathrm{H}) ;{ }^{13} \mathrm{C} \mathrm{NMR}\left(75 \mathrm{MHz}, \mathrm{CDCl}_{3}\right)$ $\delta 156.08,146.82,143.27,137.88,129.87,129.80,128.24$, $122.21,121.55,119.34,111.96,97.29,55.81$; IR $\left(\mathrm{cm}^{-1}\right) 3229$ (NH str.), 2955 (CH str.), $1624(\mathrm{C}=\mathrm{N}), 1576(\mathrm{C}=\mathrm{C}), 1160$ 
(C-O); HRMS $\left(\mathrm{ES}^{+}\right)$Calculated for $\mathrm{C}_{14} \mathrm{H}_{12} \mathrm{~N}_{2} \mathrm{BrO}[\mathrm{M}+\mathrm{H}]^{+}$: 303.0133, found 303.0146.

3-(Phenylethynyl)-5-[(trimethylsilyl)ethynyl]pyridin-2-amine 19. To a flame-dried round-bottom flask under an argon atmosphere containing 2-amino-3-iodopyridine derivative $\mathbf{1 0}$ ( $1.379 \mathrm{~g}, 4.61 \mathrm{mmol})$ was added CuI $(0.01756 \mathrm{~g}, 2 \mathrm{~mol} \%)$ and $\mathrm{Pd}\left(\mathrm{PPh}_{3}\right)_{4}(0.1066 \mathrm{~g}, 2 \mathrm{~mol} \%)$ in one portion followed by the addition of a degassed phenylacetylene solution in THF $(0.4702 \mathrm{~g}, 4.61 \mathrm{mmol})$. Triethylamine $(5 \mathrm{ml})$ was then added and the reaction mixture was stirred at rt, until no starting material was present as monitored by thin layer chromatography (TLC). After this time, the reaction was quenched with a saturated aqueous ammonium chloride and was extracted with dichloromethane. The combined organic extracts were dried over magnesium sulfate, filtered through either silica or celite and the excess solvent was removed on a rotary evaporator. The resulting crude material was taken up in dry THF $(20 \mathrm{ml})$ and treated with trimethylsilylacetylene $(5 \mathrm{ml})$, $\mathrm{Et}_{3} \mathrm{~N}(5 \mathrm{ml}), \mathrm{CuI}(17.56 \mathrm{mg}), \mathrm{Pd}\left(\mathrm{PPh}_{3}\right)_{4}(0.1066 \mathrm{~g})$ and the reaction mixture was heated to $70{ }^{\circ} \mathrm{C}$. After $8 \mathrm{~h}$ the reaction mixture was worked up as described previously, followed by purification using flash chromatography (30\% EthOAc-hexane) to afford 19 as a yellowish solid $(1.139 \mathrm{~g}, 85 \%)$. Mp: $155-158{ }^{\circ} \mathrm{C} ;{ }^{1} \mathrm{H}$ NMR $\left(300 \mathrm{MHz}, \mathrm{CDCl}_{3}\right) \delta 8.18(\mathrm{~d}, J=1.9,1 \mathrm{H}), 7.71(\mathrm{~d}, J=2.1,1 \mathrm{H})$, 7.57-7.46 (m, 2H), 7.37 (dd, $J=9.6,6.1,3 \mathrm{H}), 5.34$ (d, $J=23.2$, $2 \mathrm{H}), 0.26(\mathrm{~s}, 9 \mathrm{H}) ;{ }^{13} \mathrm{C}$ NMR $\left(75 \mathrm{MHz}, \mathrm{CDCl}_{3}\right) \delta 157.79,151.11$, 142.72, 131.56, 128.89, 128.53, 122.35, 109.63, 102.85, 101.84, 95.90, 94.99, 83.38, 0.01; IR $\left(\mathrm{cm}^{-1}\right) 3283$ (NH str.), 2956 (CH str.), 2144 (alkyne), $1625(\mathrm{C}=\mathrm{N}), 1583(\mathrm{C}=\mathrm{C})$; HRMS $\left(\mathrm{ES}^{+}\right)$ Calculated for $\mathrm{C}_{18} \mathrm{H}_{18} \mathrm{~N}_{2} \mathrm{Si}[\mathrm{M}+\mathrm{H}]^{+}$: 291.2238, found 291.2237.

2-Phenyl-5-[(trimethylsilyl)ethynyl]-1H-pyrrolo[2,3-b]pyridine 20. The forgoing 2-amino-3-ethynylpyridine derivative 19 ( $0.5513 \mathrm{~g}, 1.90 \mathrm{mmol}$ ) was dissolved in MeCN. To the resulting solution was added trifluoroacetic acid $(0.2164 \mathrm{~g}, 0.15 \mathrm{ml}$, $1.90 \mathrm{mmol}$ ) in one portion followed by the addition of trifluoroacetic anhydride $(0.5187 \mathrm{~g}, 0.35 \mathrm{ml}, 2.47 \mathrm{mmol})$ dropwise. The resulting mixture was then heated to reflux for $8 \mathrm{~h}$ and then allowed to cool to rt. The solvent was then removed on a rotary evaporator and the resulting crude material was redissolved in dichloromethane and washed with aqueous sodium carbonate $(10 \%)$. Purification by flash silica using ethyl acetate-hexane mixture (10-50\%) gave 2-phenyl-5-[(trimethylsilyl)ethynyl]-1 $H$-pyrrolo[2,3-b]pyridine $20(0.4236 \mathrm{~g}$, 91\%). Mp: 255-258 ${ }^{\circ} \mathrm{C} ;{ }^{1} \mathrm{H}$ NMR (500 MHz, $\left.\mathrm{CDCl}_{3}\right) \delta 11.56$ (s, $1 \mathrm{H}), 8.40(\mathrm{~d}, J=1.7,1 \mathrm{H}), 8.05(\mathrm{~d}, J=1.6,1 \mathrm{H}), 7.85-7.76(\mathrm{~m}$, $2 \mathrm{H}), 7.53(\mathrm{t}, J=7.7,2 \mathrm{H}), 7.47-7.38(\mathrm{~m}, 1 \mathrm{H}), 6.75(\mathrm{~d}, J=2.0$, $1 \mathrm{H}), 0.30(\mathrm{~s}, 9 \mathrm{H}) ;{ }^{13} \mathrm{C} \mathrm{NMR}\left(126 \mathrm{MHz}, \mathrm{CDCl}_{3}\right) \delta 148.74,146.08$, 140.24, 131.93, 131.78, 129.19, 128.64, 125.75, 121.46, 112.42, 103.65, 97.73, 94.57, 0.06; IR ( $\left.\mathrm{cm}^{-1}\right) 3249$ (NH str.), 2957 (CH str.), $1609(\mathrm{C}=\mathrm{N}), 1586(\mathrm{C}=\mathrm{C})$; HRMS $\left(\mathrm{ES}^{+}\right)$Calculated for $\mathrm{C}_{18} \mathrm{H}_{19} \mathrm{~N}_{2} \mathrm{Si}[\mathrm{M}+\mathrm{H}]^{+}:$291.1318, found 291.1327.

\section{Antimicrobial activity}

The substituted azaindoles were quantitatively evaluated for antimicrobial activity using the minimum inhibitory concentration (MIC) assay. ${ }^{18}$ The azaindoles were tested against a number of reference test organisms including Gram-positive (Enterococcus faecalis ATCC 29212 and Staphylococcus aureus ATCC 25923), Gram-negative (Pseudomonas aeruginosa NCTC 9027, Escherichia coli ATCC 8739 and Klebsiella pneumoniae ATCC 13883) and yeasts (Candida tropicalis ATCC 201380, Candida albicans ATCC 10231 and Cryptococcus neoformans ATCC 90112). The positive control used for the bacteria was ciprofloxacin, while that used for the yeasts was amphotericin. The solvent (acetone) was included in the assay as a negative control. All antimicrobial assays were undertaken at least in duplicate or triplicate where necessary.

\section{Acknowledgements}

This work was supported by the National Research Foundation (NRF, GUN 2053652 and IRDP of the NRF (South Africa) for financial support provided by the Research Niche Areas programme), Pretoria, and the University of the Witwatersrand (Science Faculty Research Council). We also gratefully acknowledge the NRF scarce skills programme and the CSIR scholarship scheme for generous funding to TCL. Dr R Mampa (University of the Witwatersrand) and Dr P Steenkamp (CSIR, Pretoria) are thanked for providing the NMR and HRMS MS spectroscopy services, respectively.

\section{Notes and references}

1 S. R. Walker, E. J. Carter, B. C. Huff and J. C. Morris, Chem. Rev., 2009, 109, 3080-3098.

2 M. Prudhomme, Eur. J. Med. Chem., 2003, 38, 123-140.

3 B. Nowicka-Sans, Y.-F. Gong, B. McAuliffe, I. Dicker, H.-T. Ho, N. Zhou, B. Eggers, P.-F. Lin, N. Ray, M. WindRotolo, L. Zhu, A. Majumdar, D. Stock, M. Lataillade, G. J. Hanna, J. D. Matiskella, Y. Ueda, T. Wang, J. F. Kadow, N. A. Meanwell and M. Krystal, Antimicrob. Agents Chemother., 2012, 56, 3498-3507.

4 (a) J. Y. Mérour, S. Routier, F. Suzenet and B. Joseph, Tetrahedron, 2013, 69, 4767-4834; (b) J. J. Song, J. T. Reeves, F. Gallou, Z. Tan, N. K. Yee and C. H. Senanayake, Chem. Soc. Rev., 2007, 36, 1120-1132; (c) J.-Y. Mérour and B. Joseph, Curr. Org. Chem., 2001, 5, 471-506; (d) F. Popowycz, S. Routier, B. Joseph and J.-Y. Mérour, Tetrahedron, 2007, 63, 1031-1064.

5 (a) M. C. de Mattos, S. Alatorre-Santamaría, V. GotorFernández and V. Gotor, Synthesis, 2007, 2149-2152; (b) C. Koradin, W. Dohle, A. L. Rodriguez, B. Schmid and P. Knochel, Tetrahedron, 2003, 59, 1571-1587; (c) M. McLaughlin, M. Palucki and I. W. Davies, Org. Lett., 2006, 8, 3307-3310; (d) A. L. Rodriguez, C. Koradin, W. Dohle and P. Knochel, Angew. Chem., Int. Ed., 2000, 39, 2488-2490; (e) C. Harcken, Y. Ward, D. Thomson and D. Riether, Synlett, 2005, 3121-3125; $(f)$ K. C. Majumdar and S. Mondal, Tetrahedron Lett., 2007, 48, 6951-6953.

6 (a) S. E. Pearson and S. Nandan, Synthesis, 2005, 25032506; (b) N. Sakai, N. Annaka, A. Fujita, A. Sato and 
T. Konakahara, J. Org. Chem., 2008, 73, 4160-4165; (c) K. C. Majumdar, S. Samanta and B. Chattopadhyay, Tetrahedron Lett., 2008, 49, 7213-7216; (d) S. Cacchi, G. Fabrizi and L. M. Parisi, J. Comb. Chem., 2005, 7, 510-512.

7 (a) A. Carpita and A. Ribecai, Tetrahedron Lett., 2009, 50, 6877-6881; (b) A. Carpita, A. Ribecai and P. Stabile, Tetrahedron, 2010, 66, 7169-7178.

8 T. C. Leboho, J. P. Michael, W. A. L. van Otterlo, S. F. van Vuuren and C. B. de Koning, Bioorg. Med. Chem. Lett., 2009, 19, 4948-4951.

9 L. V. Politanskaya, I. P. Chuikov, E. A. Kolodina, M. S. Shvartsberg and V. D. Shteingarts, J. Fluorine Chem., 2012, 135, 97-107.

10 K. Venkateswarlu, K. Suneel, B. Das, K. N. Reddy and T. S. Reddy, Synth. Commun., 2009, 39, 215-219.

11 K. Kumaran, M. K. S. Raja and K. R. Jaisankar, Int. J. PharmTech Res., 2012, 4, 169-175.

12 N. Lebouvier, F. Pagniez, M. Duflos, P. Le Pape, Y. M. Na, G. Le Baut and M. Le Borgne, Bioorg. Med. Chem. Lett., 2007, 17, 3686-3689.
13 R. M. La Hoz and P. G. Pappas, Drugs, 2013, 73, 495504.

14 Y. Liao, M. Chen, T. Hartmann, R. Y. Yang and W. Q. Liao, Chin. Med. J., 2013, 126, 361-368.

15 H.-R. Tsou, N. Mamuya, B. D. Johnson, M. F. Reich, B. C. Gruber, F. Ye, R. Nilakantan, R. Shen, C. Discafani, R. DeBlanc, R. Davis, F. E. Koehn, L. M. Greenberger, Y.-F. Wang and A. Wissner, J. Med. Chem., 2001, 44, 27192734.

16 G. Acquaah-Harrison, S. Zhou, J. V. Hines and S. C. Bergmeier, J. Comb. Chem., 2010, 12, 491-496.

17 B. Cash, C. Fischer, Y. Garcia, J. Jung, J. Katz, J. Kim, A. Rivkin, A. Schell, T. Siu, D. Witter and H. Zhou, Azaindoles as janus kinase inhibitors, WO 2011137022 A1, 2011, 3 November 2011.

18 CLSI, Clinical and Laboratory Standards Institute, Methods for Dilution Antimicrobial Susceptibility Tests for Bacteria that Grow Aerobically, Approved Standard M100-S22, National Committee for Clinical Laboratory Standards, Fort Wayne, Ind, USA, 22nd edn, 2012. 